\title{
The Fault Location Method Research of Three-Layer Network System
}

\author{
Hu Shaolin ${ }^{1,2}$, Li ye $^{2}$, Karl Meinke ${ }^{3}$ \\ (1. State Key Laboratory of Astronautics, Xi'an, P.O. Box 505-16, 710043,China) \\ (2. Automation School, Xi'an University of Technology, Xi'an, 230027) \\ (3. Royal Technical Institute of Sweden, Stochastic, Sweden)
}

\begin{abstract}
The fault location technology research of three-layer network system structure dynamic has important theoretic value and apparent engineering application value on exploring the fault detection and localization of the complex structure dynamic system. In this article, the method of failure propagation and adverse inference are adopted, the fault location algorithm of the three-layer structure dynamic network system is established on the basis of the concept of association matrix and the calculating method are proposed, and the simulation calculation confirmed the reliability of this paper. The results of the research can be used for the fault diagnosis of the hierarchical control system. testing of the engineering software and the analysis of the failure effects of layered network of all kinds and other different fields.
\end{abstract}

Keywords- Three-layer network; fault propagation; fault location.

\section{INTRODUCTION}

Nearly half a century, the automation technology of system during operating process has developed along two general directions as a whole. One is the optimization and intelligent of the system in normal conditions, the other is fault monitoring and safety of the system in failure. Especially the latter, since the 1970s, Berd put forward a control system which is based on the analytic redundant fault detection and diagnosis, it was widely valued by the international and domestic academic industry and the engineering application industry, successively proposed and developed a series of fault detection and diagnosis

methods that are novel and practical (FDD : Fault Detection and Diagnosis), such as FDD method based on signal processing FDD method based on data-driven, FDD method based on structural redundancy, FDD method based on information redundancy , method based on equivalent space and FDD method based on system simulation etc. The concerns of the research have developed from early static equipment or the single fault of the system to the concurrent malfunction of the complex structure system and the continual failure of the system during running process.

For the fault monitoring and security of a complicated structure dynamic system, it has been the focus and difficulty that how to use the anomaly information monitored by all sorts of sensing/sensors or other measuring equipment to fault location accurately.

Complex structure dynamic system has a complex relationship between relevant components, the difficulty of the fault location become higher and higher, in order to overcome the technology difficulty of the develop of the fault diagnosis expert system and the technical problems of familiar reasoning process conflicts which are based on rule reasoning or cases, this paper develops a practical and concise fault location algorithm based on mathematical operation, which can locate the fault point fastly, it also solves the difficult problems of fault location of the complex structure system and proves the effectiveness of the new method through the simulation results.

\section{ThE Status ANALYSIS OF COMPLEX STRUCTURE SYSTEM AND CAUSAL MODELING}

Any complex structure dynamic system is usually composed of several functional subsystem, each subsystem includes several components. Similarly, each subsystem can also be further broken down into some functional subsystem in level 2 and each subsystem in level 2 also includes several small parts.

Whether the systems or the subsystems or the components, only when it transfer information to the outside system directly or indirectly, can we perceive or judge whether it runs normal or not. In other words, whether the state of work is normal or not is unknown to the outside world without any way to transform subsystems or components to the outside system directly or indirectly. For this kind of system that cannot transfer parts of information or subsystems to the outside system, we call it a "black hole".

For this kind of system, when malfunction happened, how to locate fault source becomes difficult, considering the complexity of the system, causal modeling method is adopted in this paper, namely layered network modeling method, it sets up the correlation matrix through the top and the bottom of the association. So, the changes of the bottom can find the changes of the top source through the incidence matrix.

\section{The Design And CAlculation OF The ToP - BOTTOM RELATING MATRIX}

For the three layer network in figure 1, simple to set the top nodes as $V_{1}=\left\{v_{1, i} \mid i=1,2, \cdots, n_{1}\right\}$, to set the middle nodes as $V_{2}=\left\{v_{2, i} \mid i=1,2, \cdots, n_{2}\right\} \quad$,to set the bottom nodes as $V_{3}=\left\{v_{3, i} \mid i=1,2, \cdots, n_{3}\right\}$, 


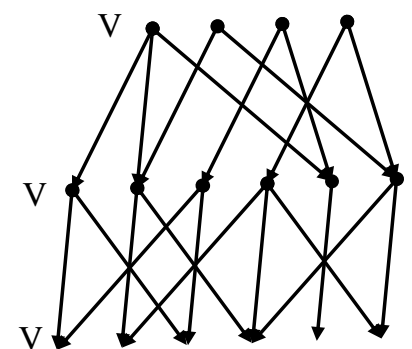

Figure 1. The structure schematic drawing of three layer network

For the bottom nodes $v_{1, j} \in V_{1}$, defining status symbol values for nodes

$$
d_{1}\left(v_{1, j}\right)=\left(\begin{array}{l}
1, \text { node } v_{1, j} \text { is fault } \\
0, \text { node } v_{1, j} \text { is normal }
\end{array}\right.
$$

And the joint strength between adjacent layer contacts nodes $v_{s, i} \in V_{s}$ and $v_{s+1, j} \in V_{s+1}$

$$
b\left(v_{s, t}, v_{s+1, j}\right)=\left\{\begin{array}{ll}
1, & v_{s, j} \text { and } v_{s+1, j} \text { has } \\
& \text { edge-juction } \\
0, & v_{s, j} \text { and } v_{s+1, j} \text { has } \\
& \text { not edge-juction }
\end{array} \quad(s=1,2)\right.
$$

Thus, using operator "and" $\wedge$ and operator "or" $\vee$ of Borel algebra, we can calculate the flag values of the middle layer nodes $v_{2, j} \in V_{2}$ by the next formula

$$
d_{2}\left(v_{2, j}\right)=\underset{t=1}{\vee}\left\{d_{1}\left(v_{1, t}\right) \wedge b\left(v_{1, t}, v_{2, j}\right)\right\}
$$

Similarly, defining the flag values of the bottom nodes $v_{3, j} \in V_{3}$

$$
d_{3}\left(v_{3, j}\right)=\underset{t=1}{\vee}\left\{d_{2}\left(v_{2, t}\right) \wedge b\left(v_{2, t}, v_{3, j}\right)\right\}
$$

Proposition 1: if $d_{2}\left(v_{2, j}\right)=0$, so either every top layer node does not broke down or the node which brokes down does not spread into the middle layer node $v_{2, j} \in V_{2}$, conversely, if $d_{2}\left(v_{2, j}\right)=1$, so the top layer has at least one node broke down, and the failure influence spread into the middle layer nodes $v_{2, j} \in V_{2}$.

Proof. By formula (4) and the operation rule of Borel operator, if $d_{2}\left(v_{2, j}\right)=0$, so for all the top layer nodes $v_{1, t} \in V_{1}$, we can have $d_{1}\left(v_{1, t}\right)=0$ or $b\left(v_{1, t}, v_{2, j}\right)=0$, it means that either this node does not broke down or there is no united way between this node and the middle layer node.
If $d_{2}\left(v_{2, j}\right)=1$, so there must be $1 \leq t_{0} \leq n_{1}$ to make $d_{1}\left(v_{1, t_{0}}\right) \wedge b\left(v_{1, t_{0}}, v_{2, j}\right)=1$,

it means $d_{1}\left(v_{1, t_{0}}\right)=1$ and $b\left(v_{1, t_{0}}, v_{2, j}\right)=1$.

Thus, the top layer node $v_{1, t_{0}}$ has broken down and its fault can transfer to middle node $v_{2, j}$ by the path $\left(v_{1, t_{0}}, v_{2, j}\right)$.

Proposition 2: if $d_{3}\left(v_{3, j}\right)=0$, so the middle layer node either works properly or the influence of the abnormal node do not transfer to the bottom layer node $v_{3, j} \in V_{3}$, reversely, if $d_{3}\left(v_{3, j}\right)=1$, so there is at least one top middle node is abnormal and its fault influence transfer to the bottom layer node $v_{3, j} \in V_{3}$.

Proof. It is familiar with proposition 1, so omit the proof process here. Making flag vector

$$
\vec{d}_{1}=\left(d_{1}\left(v_{1,1}\right), \cdots, d_{1}\left(v_{1, n 1}\right)\right),
$$

setting the first $\mathrm{k}$ component of vector to 1 , the rest is 0 respectively.

$$
\begin{gathered}
\vec{d}_{1}{ }^{k}=(1, \cdots, 1, \cdots, 0) \\
\uparrow
\end{gathered}
$$

\section{the first $k$ component}

Using the flag value of the middle layer node $d_{2}^{k}\left(v_{2, j}\right) \quad\left(j=1, \cdots, n_{2}\right)$ can calculate the numbers of the propagation paths that the top layer node uses to transfer the fault to the bottom layer node.

$$
s_{i}(k)=\sum_{j=1}^{n 2} d_{2}^{k}\left(v_{2, j}\right) \wedge b\left(v_{2, j}, v_{3, i}\right)
$$

According to the definition of the formula (6) and the proving process of propoistion 1 and proposition 2, we can easily derive the next conclusion:

Theorem 1: if $s_{i}(k)=t$, showing that the top layer node $v_{1, k}$ is broken down and it will transfer its fault to the bottom layer node $v_{3, i}$ through $\mathrm{t}$ different paths.

If $s_{i}(k)=0$, it shows that the fault of the top layer node $v_{1, k}$ do not effect the bottom layer node $v_{3, i}$.

Proof: according to formular (6) and the operational rule of Borel operator, we can find that if $d_{2}^{k}\left(v_{2, j}\right) \wedge b\left(v_{2, j}, v_{3, i}\right)=1$, it means $d_{2}^{k}\left(v_{2, j}\right)=1$ and $b\left(v_{2, j}, v_{3, i}\right)=1$. Thus, the top layer node $v_{1, k}$ is borken down and it can transfer its fault from the 
middle layer node $v_{2, j}$ to the bottom layer node $v_{3, i}$ by the path $\left(v_{2, j}, v_{3, i}\right)$.

Thus, the formula (6) shows the numbers of paths that top layer node uses to transfer its fault form different middle layer nodes to the bottom layer nodes.

Obviously, if

$$
\sum_{i=1}^{n_{3}} s_{i}(k)=0, \text { So, } s_{1}(k)=\cdots=s_{n 3}(k)=0 \text {. }
$$

According to the proposition 3, the fault of the first $\mathrm{k}$ layer node can not be transferred and can not effect the bottom layer node. We called these top layer nodes isolated points.

For the three-layer network system without isolated points, we can eastablish a top-bottom associated matrix by calculating the numbers of the paths $s_{i}(k)$ that the top layer node uses to transfer its fault to the bottom layer nodes.

$$
M_{1-3}=\left(\begin{array}{ccc}
v_{3,1} & \cdots & v_{3, n 3} \\
f_{1,1} & \cdots & f_{1, n 3} \\
\vdots & & \vdots \\
f_{n 1,1} & \cdots & f_{n 1, n 3}
\end{array}\right) v_{1,1}
$$

In the formula (6)

$$
f_{k, i}=s_{i}(k) / \sum_{i=1}^{n 3} s_{i}(k) \quad\left(k=1, \cdots, n_{1} ; i=1, \cdots, n_{3}\right)
$$

Obviously, the matrix $M_{1-3}$ has the next qualities:

(1) $0 \leq f_{k, i} \leq 1 \quad\left(k=1, \cdots, n_{1} ; i=1, \cdots, n_{3}\right)$, it means every elements of the associated matrix is a nonnegative number that is not exceeding 1 .

(2) $\sum_{j=1}^{n_{3}} f_{k, i}=1 \quad\left(k=1, \cdots, n_{1}\right)$, it means that all elements in each row of the matrix added to 1 .

\section{THE AlgORRTHM OF THE FAUlt LOCATION}

Bringing all different groups of the formular (5) into the formular (3) to calculate the flag value $d_{2}^{k}\left(v_{2, j}\right) \quad\left(j=1, \cdots, n_{2}\right)$ using proposition 1 and proposition 2 , then bringing the flag value $d_{2}^{k}\left(v_{2, j}\right)$

into the formular (4), after the above process, we can calculate the state flag matrix:

$$
B_{1-3}=\left(\begin{array}{ccc}
\vdots v_{3,1} & \cdots \cdots & v_{3, n 3} \\
d_{3}^{1}\left(v_{3,1}\right) & \cdots & d_{3}^{1}\left(v_{3, n 3}\right) \\
\vdots & & \vdots \\
d_{3}^{n 1}\left(v_{3,1}\right) & \cdots & d_{3}^{n 1}\left(v_{3, n 3}\right)
\end{array}\right) v_{1,1}
$$

Obviously, the flag value $d_{3}^{i}\left(v_{3, j}\right)$ of i-th $\left(i=1,2, \cdots, n_{1}\right)$ row and the $\mathrm{j}$-th $\left(j=1,2, \cdots, n_{3}\right)$ in the state flag matrix $B_{1-3}$ reflect whether the top layer node $v_{1, i}$ can transfer its fault to the bottom layer node $v_{3, j}$, reversely, $d_{3}^{i}\left(v_{3, j}\right)=0$,shows that the bottom layer node $v_{3, j}$ would not be effected by the top layer node $v_{1, i}$.

According to the analysis above, we can establish the fault location algorithm: we supposed that every bottom layer node is installed with a sensor, so we can achieve the fault omen information of the layer node directly. According to the state information of the bottom layer of the system, we can demarcate the state of the bottom layer nodes

$$
f_{3}\left(v_{3, j}\right)=\left(\begin{array}{l}
1, \text { node } v_{3, j} \text { has fault omen } \\
0, \text { node } v_{3, j} \text { is normal }
\end{array}\right.
$$

Calculating the weighting indicator:

$$
g_{i}=\sum_{j=1}^{n_{3}} f_{i, j}\left\{d_{3}^{i}\left(v_{3, j}\right) \wedge f_{3}\left(v_{3, j}\right)\right\}\left(i=1,2, \cdots, n_{1}\right)
$$

It is easy to prove $0 \leq g_{i} \leq 1\left(i=1,2, \cdots, n_{1}\right)$. When $g_{i}=1$, we can diagnose the top layer node $v_{1, i}$ is the source of the fault, when $g_{i}<1$, we can diagnose the top layer node $v_{1, i}$ is normal (or the fault omens of the bottom layer nodes are not caused by the top layer node $v_{1, i}$ ).

In practical operation, considering the fault omen or the sensitivity of the detection sensor, when $g_{i} \approx 1$ and $g_{i}<<1$, we can make the similar judge as above.

\section{Simulation CALCUlation}

For the three-layer network as the figure 1 shows, we use $4 \times 4$ matrix

$$
D 1=\left[\begin{array}{llll}
1 & 0 & 0 & 0 \\
0 & 1 & 0 & 0 \\
0 & 0 & 1 & 0 \\
0 & 0 & 0 & 1
\end{array}\right]
$$

to express all the groups of the formula (5), comparing the matrix $\mathrm{D} 1$, we assume the fault nodes are $v_{1,1}$ and $v_{1,3}$, according to the figure 1 , we can calculate the flag matrix of the bottom layer 


$$
f=\left[\begin{array}{llllll}
1 & 0 & 1 & 0 & 1 & 0
\end{array}\right] .
$$

Then, calculating the state flag matrix when the top layer nodes transfer its faults to the bottom layer nodes by the formulae (3) and (4)

$$
D 3=\left[\begin{array}{llll}
1 & 1 & 1 & 1 \\
0 & 1 & 0 & 1 \\
1 & 0 & 1 & 0 \\
0 & 1 & 0 & 1
\end{array}\right],
$$

using $f_{k, i}=\frac{s_{i}(k)}{\sum_{i=1}^{n 3} s_{i}(k)} \quad\left(k=1, \cdots, n_{1} ; i=1, \cdots, n_{3}\right)$ to help

us to get the weighting matrix, it is also the top-bottom associated matrix

$$
F=\left[\begin{array}{cccc}
0.2 & 0.2 & 0.2 & 0.2 \\
0 & 0.3333 & 0 & 0.3333 \\
0.3333 & 0 & 0.3333 & 0 \\
0 & 0.25 & 0 & 0.25
\end{array}\right] \text {, }
$$

finally, we can get the weighting indicator matrix of the top layer nodes by using the formula (10)

$$
g=\left[\begin{array}{llll}
0.6 & 0 & 1 & 0
\end{array}\right]
$$

.We can make the conclusion from the simulation result of matlab that $\mathrm{g}(1,1)$ and $\mathrm{g}(1,3)$ satisfy the condition $g_{i} \approx 1$, so we can judge the nodes $v_{1,1}$ and $v_{1,3}$ are the fault nodes, it proves the accuracy of the algorithm.

\section{ACKNOWLEDGMENT}

The authors wish to express special thank to the financial support of the National Natural Science Foundation of China, Grant No. 61074077.

\section{CONCLUSION}

It is necessary to detect and protect the state of the complex structure system in the engineering field, how to detect and repair timely when faults happen to the complex structure system is more important. For the complex system which concludes many different functional subsystems, how to locate the source of the faults quickly is the key point of this paper. The paper set a example as the three-layer network, putting forward a new algorithm of fault location that can locate the source of fault by the detection of sensors to the bottom layer nodes, it can save much time for the next work. In the last of the article, we make a simulation test to the algorithm using the software matlab, the result prove the accuracy of the algorithm. Also, the algorithm is only tested in the three-layer network system, but we cannot get the accuracy result for the more complex system and it will be the main direction of the research in the future.

\section{Reference}

[1] Zhang Yingwei, S.Joe Qin. The Fault Diagnosis of the Complex Industry Process [in chinese]. Journal of Northeast University Press, Shenyang, 2008

[2] Zhou Donghua, Sun Youxian. Fault Detection and Diagnosis Techonlogy of the Control System [in chinese]. Tsinghua University Press, Beijing, 1994

[3] Lijing, Liu Hongwei, Dongjian, Shu Yanjun. The Cluster Fault Management System Structure Based On the Independent Calculation [in chinese]. Journal of Tsinghua University, 2011, $\mathrm{S} 1: 1-5$

[4] Heidtmann, F. Soffker, D. Virtual Sensors for Diagosis and Prognosis Purposes in the Context of Elastic Mechanical Structures. Sensors Journal, IEEE,2009,9(11):1577-1588

[5] Lira, D.N. Garcia, J.1. Junqueira, F. Miyagi, P.E. Fault detection in Flexible Assembly Systems using Petri net. Latin America Transaction, IEEE, 2008,6(7):572-578

[6] Xiong Hao, Sun Cai-xin: Arificial Immune Network Classification Algorithm for Fault Diagonsis of Power Transformei. Power Delivery, IEEE Transactions, 2007, 22(2):(930-935)

[7] Dorel Arordachioaie, Emil Ceangaa, et al. Detection and classification of non-linearities based on Volterra kernels processing. Engineering Applications of Artificial Intelligence,The International Journal of Intelligent Real-Time Automation,2001,14(4): 497-503

[8] Xue Dingyu: The Simulation Technology and Application of the System Based on Matlab/Simulink [in chinese].Tsinghua University Press, Beijing, 2011 